\title{
Ultra Fast and Highly Realistic Numerical Modelling of the Surface EMG
}

\author{
Kostiantyn Maksymenko ${ }^{1}$, Samuel Deslauriers-Gauthier ${ }^{1,2}$, and \\ Dario Farina ${ }^{3}$ \\ ${ }^{1}$ Neurodec, Sophia Antipolis, France \\ ${ }^{2}$ Inria Sophia Antipolis - Méditerranée, Université Côte D’Azur, \\ Nice, France \\ ${ }^{3}$ Department of Bioengineering, Imperial College London, London, \\ UK
}

June 7, 2021

\begin{abstract}
Modelling the biophysics underlying the generation and recording of electromyographic (EMG) signals has had a fundamental role in our understanding of muscle electrophysiology as well as in the validation of algorithms for information extraction from the EMG. Current EMG models differ for the complexity of the description of the volume conductor. Analytical solutions are computationally efficient for a small number of fibers but limited to simplified geometries. Numerical solutions are based on accurate anatomical descriptions but require long computational time and are therefore impractical for applications requiring a large number of simulations across a broad variety of conditions. Here, we propose a computationally efficient and realistic EMG model. The volume conductor is described from magnetic resonance images (MRI) or tissue surfaces by discretization in a tetrahedral mesh. The numerical solution of the forward model is optimized by reducing the main calculations to the solutions in a minimal number of basis points, from which the general solution can be obtained. This approach allows the lowest computational time than any current EMG models and also provides a scalable solution. New solutions for the same volume conductor can indeed be obtained without recomputing the volume conductor transformation. This property provides almost real-time simulations, without any constraints on the complexity of the volume conductor or of the transmembrane current source. Because of the high computational efficiency, the proposed model can be used as a basis for the solution of the inverse model or as a means to simulate a large number of data for artificial intelligence (AI) based EMG processing.
\end{abstract}




\section{Introduction}

Biophysical modelling of the generation and recording of muscle electrical signals (electromyography, EMG) has been extensively described (for review, see [1]). The solution of the forward problem in EMG generation is based on Maxwell's equations. However, as for other biosignals, because of the relatively low frequencies of the sources, a quasi-static assumption can be applied 2, 3. This assumption simplifies the problem to the solution of the Poisson equation 2, 4, 5, with Neumann boundary conditions. With this simplification, the forward problem can be analytically solved for specific geometries of the volume conductor, such as for the planar (e.g., [6]) or cylindrical (7, 8]) shapes. Accordingly, analytical EMG models based on these geometries have been developed and extensively used (e.g., 9, 10, 11, 12, 13]).

The simple geometries treated with analytical solutions provide simulations which reflect the broad characteristics of EMG signals but cannot be used to interpret specific experimental conditions or to reverse the model for source identification. More realistic models of EMG generation are based on numerical solutions of the Poisson equation with generic volume conductor shapes (e.g., 14, 15]). Nonetheless, numerical EMG models have had so far limited applicability because of the high computational time they require. Currently, there are no computationally efficient models of EMG generation with highly accurate description of the volume conductor. Therefore, the use of EMG models is not extensive. They are mainly applied to identify the generic associations between physiological mechanisms and features of the EMG signal (e.g., [10, 12).

Because of the recent breakthroughs in artificial intelligence in association with decoding surface EMG signals into individual motor unit activities [16, 17, the availability of highly accurate EMG models has become of renewed importance. Highly accurate models with computational speed comparable to simplified analytical models would allow addressing the decoding problem for EMG by providing arbitrarily large sets of data for training deep neural networks. Moreover, these models would find potential applications in developing inverse solutions for source identification [18. The combination of almost real-time precise modelling and artificial intelligence would open new perspectives in the use of EMG for building neuromuscular human-machine interfaces, for diagnostics, and for neuroscientific investigations.

Here we describe an EMG model based on the numerical solution of the volume conductor equations. The forward solutions are computed for selected point current sources (basis sources) and the response of the system to any current source inside the volume conductor is calculated as combination of the solutions of the basis sources. With this approach, we show that it is possible to generate simulated EMG signals from several thousands of muscle fibers within very limited computation time (in the order of seconds). Moreover, each element of the model is independent so that only the model features that are changed in each simulation need to be re-computed. This characteristics allows achieving realtime simulations. The model allows breakthrough approaches in EMG inverse modelling and AI-based EMG decoding. Validation of the model by comparison 


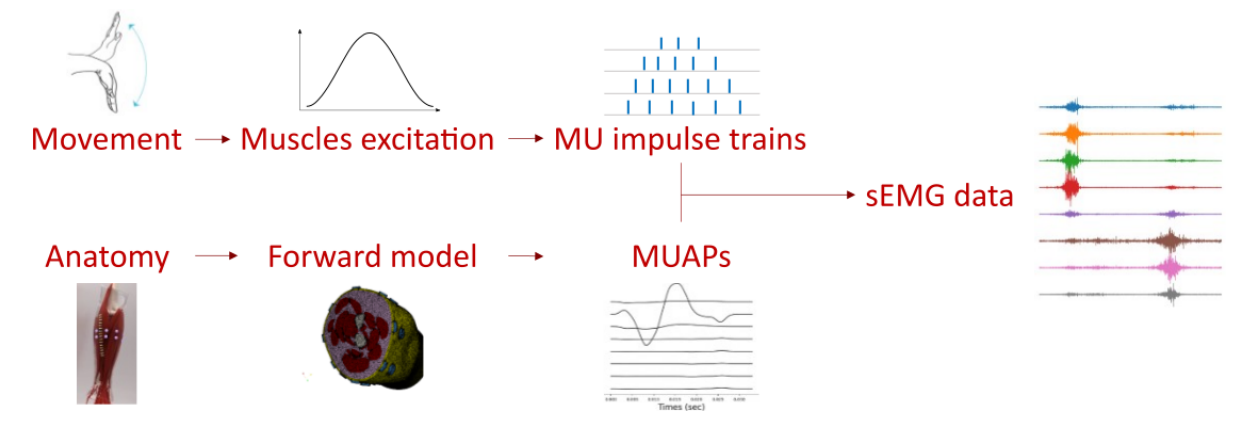

Figure 1: The general pipeline of sEMG simulation. The main steps include: 1) Motor units (MUs) recruitment model, i.e. decomposing muscle excitation into MU impulse trains; 2) Forward problem, i.e. using conductor, electrode and source models to simulate MU action potentials (MUAPs); 3) Combining MU impulse trains with corresponding MUAPs to obtain the simulated sEMG signal.

with analytical solutions as well as representative applications of the model are also described.

\section{Methods}

In this section we present the methods which allow the implementation of a realistic and near real-time surface EMG (sEMG) simulator (Fig. 1). First, we cover in detail an efficient strategy to solve the EMG forward problem and discuss its implementation. Second, we present methods to generate muscle fibers and motor units (MUs) from surface meshes, MU action potentials (MUAPs) and the MU recruitment model (decomposing muscle excitation into MU impulse trains). Together, these tools allow the realistic modeling of muscle physiology and associated sEMG signals from a straightforward model description.

\subsection{Forward problem}

The fiber extracellular potentials that are measured by EMG electrodes are generated by transmembrane currents. The properties of bioelectric currents and potential fields can be determined from solutions of the Maxwell's equations, taking into account the electrical properties of biological tissues. Because of the relatively low frequencies of signal sources of biological origin, the quasi-static assumption can be applied 2, 3, so that the electric potential and the primary current sources are related by the following Poisson equation [2, 4, 5] with Neumann boundary conditions: 


$$
\left\{\begin{array}{l}
\nabla \cdot(\sigma \nabla \phi)=-I \quad \text { in } \Omega \\
\sigma \frac{\partial \phi}{\partial \boldsymbol{n}}=\sigma \nabla \phi \cdot \boldsymbol{n}=0 \quad \text { on } \partial \Omega
\end{array}\right.
$$

where $\Omega \subset \mathbb{R}^{3}$ is a volume conductor domain of interest, $\partial \Omega$ its boundary with outward pointing normal unit vector $\boldsymbol{n}, \phi(\boldsymbol{r})[V]$ is the electric potential, $I(\boldsymbol{r})\left[A / m^{3}\right]$ is the current source density (CSD), $\sigma(\boldsymbol{r})[S / m]$ is a conductivity tensor. The second line of the equation (boundary condition) reflects the assumption that no current flows out of the domain of interest. In the context of EMG modeling, this implies that there is no current flow between the skin and air. The current source density $I(\boldsymbol{r})$ is interpreted as the volume density of current entering or leaving the extracellular medium at position $\boldsymbol{r} \in \Omega$. A negative CSD corresponds to current leaving the extracellular medium (due to the fiber transmembrane currents) and is thus conventionally called a sink. Likewise, current entering the extracellular medium is called a source [19, 20].

Equation (1) cannot be solved analytically for general volume conductor geometries, but several numerical methods can be used to approximate its solution. Here, we use the finite element method (FEM) [21, which discretizes the volume conductor $\Omega$ as a tetrahedral mesh $\Omega_{t}$. Given this mesh, we use the Galerkin method to project the potential $\phi$ onto the space of piecewise affine functions defined on $\Omega_{t}$. Fig. 2 illustrates an example of a discretized mesh representing a realistic forearm model.

This discretization process converts the continuous operator problem of Eq. (1) to a finite system of linear equations:

$$
A \boldsymbol{v}=\boldsymbol{b}
$$

where $A$ is a symmetric and sparse $n_{v} \times n_{v}$ matrix, $n_{v}$ is the number of mesh vertices, $\boldsymbol{v} \in \mathbb{R}^{n_{v}}$ is a vector of potential values at mesh nodes, and $\boldsymbol{b} \in \mathbb{R}^{n_{v}}$ is a vector containing source information. Because the electric potential is defined up to a constant, the matrix $A$ always has a one dimensional null space. To obtain a unique solution to the system of Eq. (2), we constrain potentials $\boldsymbol{v}$ to have a zero sum.

In the context of EMG, we are not interested in finding electric potentials everywhere in the conductor, but only at the electrode locations. Let $S$ be a selection matrix with a shape $n_{e} \times n_{v}$ which only selects the values at EMG electrode locations ( $n_{e}$ is the number of electrodes). Each row of $S$ can be designed to select a single point location or to integrate over an area (e.g. the electrode-skin interface) depending on the location and number of its non-zero elements. Also, let $\boldsymbol{b}(\boldsymbol{r})$ correspond to a point source at location $\boldsymbol{r}$. The resulting EMG signal is thus given by:

$$
\boldsymbol{v}_{\text {point }}(\boldsymbol{r})=S A^{-1} \boldsymbol{b}(\boldsymbol{r}) .
$$




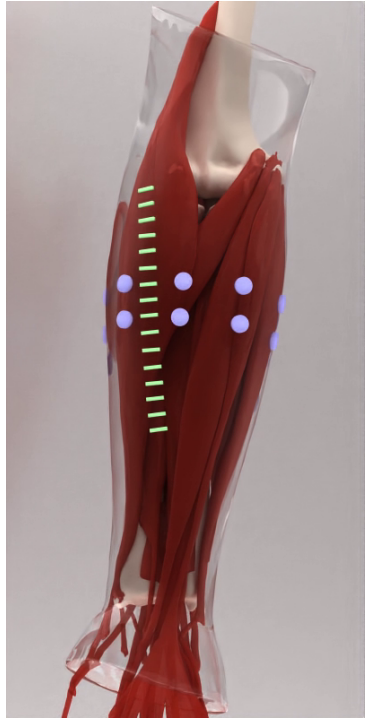

(a)

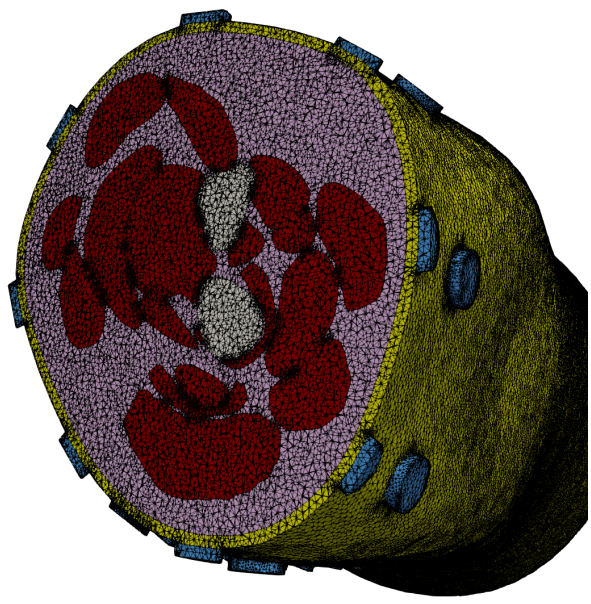

(b)

Figure 2: (a) Surface geometry of muscles, bones, subcutaneous tissue, skin and electrodes used for arm modeling (taken from BodyParts3D, The Database Center for Life Science [22]). (b) Cross section of the volume mesh $\Omega_{t}$ generated from the arm surfaces.

\subsubsection{Efficient implementation}

Let us analyze in more detail the structure of $A$ and $\boldsymbol{b}$ from Eq. (2). Let $\left\{w^{i}(\boldsymbol{r}), i=1 \ldots n_{v}\right\}$ be a set of $n_{v} P^{1}$ (piecewise linear) basis functions over the tetrahedral mesh $\Omega_{t}$. Note, that $w^{i}$ is 1 at the $i$-th vertex of the mesh, is 0 at all other vertices and is linear at all tetrahedra adjacent to the $i$-th vertex. In this case $A$ and $\boldsymbol{b}$ have the following structure:

$$
\begin{aligned}
& A_{i j}=\int_{\Omega_{t}} \sigma(\boldsymbol{r}) \nabla w^{i}(\boldsymbol{r}) \nabla w^{j}(\boldsymbol{r}) d \boldsymbol{r} \\
& b_{i}=\int_{\Omega_{t}} I(\boldsymbol{r}) w^{i}(\boldsymbol{r}) d \boldsymbol{r} .
\end{aligned}
$$

First, let us notice that $A$ is symmetric and, in general, a very large matrix which can be stored only because it is sparse. Indeed, the functions $w^{i}$ have a compact support and their pairwise scalar product is non-zero only for "neighbor" functions. Since the pseudo-inverse (or the inverse) of a sparse matrix is usually not a sparse matrix, it is impractical to compute it because of the amount of memory needed to store it. Thus, iterative methods are typically used to solve the system of Eq. (2) for every given $\boldsymbol{b}$. 
Consider the case of $I(\boldsymbol{r})=\delta(\boldsymbol{r}-\overline{\boldsymbol{r}})$ which corresponds to a unit point current source at location $\overline{\boldsymbol{r}}$. Without loss of generality, we assume that this source is inside a tetrahedron formed by the vertices $i_{1}, \ldots, i_{4}$ of the mesh. In this case, we obtain:

$$
b_{i}=\left\{\begin{array}{l}
\lambda_{j}, \text { if } i \in\left\{i_{1}, \ldots, i_{4}\right\} \\
0, \text { otherwise }
\end{array}\right.
$$

where $\left\{\lambda_{j}, j=1, \ldots, 4\right\}$ are the barycentric coordinates of the point $\overline{\boldsymbol{r}}$ inside the tetrahedron $\left\{i_{1}, \ldots, i_{4}\right\}$. Applying this expression to Eq. (3), we obtain:

$$
\boldsymbol{v}_{\text {point }}(\overline{\boldsymbol{r}})=S A^{-1} \boldsymbol{b}(\overline{\boldsymbol{r}})=S A^{-1} \bar{B} \boldsymbol{\lambda} .
$$

where $\bar{B}$ is a $n_{v} \times 4$ matrix with $\bar{B}_{i_{j}, j}=1$ for $j=1, \ldots, 4$, and 0 otherwise. This implies that the solution of the system of Eq. (2) for any unit point source can be computed as a barycentric sum of solutions on the vertices of the corresponding tetrahedron. Therefore, it is sufficient to compute solutions of Eq. (2) for "basis" sources located on mesh vertices, to be able to evaluate a solution for any point inside this mesh in an efficient way. Let $n_{s}$ be the number of such basis sources. For the most general case, when the source can be located anywhere inside the mesh and $n_{s}=n_{v}$, let $B$ be a $n_{v} \times n_{s}$ identity matrix. The objective is to compute "basis" solutions:

$$
V_{\text {basis }}=S A^{-1} B
$$

where $V_{b a s i s}$ is a $n_{e} \times n_{s}$ matrix, whose columns contain the solutions of Eq. (2) for a unit point source located at the corresponding mesh vertex. Hence, the potentials for any source location $\boldsymbol{r}$ is given by:

$$
\boldsymbol{v}_{\text {point }}(\boldsymbol{r})=V_{\text {basis }} \boldsymbol{\lambda}(\boldsymbol{r})
$$

where $\boldsymbol{\lambda}(\boldsymbol{r}) \in \mathbb{R}^{n_{s}}$ is a vector, whose four non-zero elements contain the barycentric coordinates of point $\boldsymbol{r}$ inside a corresponding tetrahedron. Note, that one may restrict potential sources to be located inside specific subdomains of the whole mesh (which is the case for EMG). In this case, $n_{s}$ corresponds to the number of vertices of these subdomains, and the matrix $B$ is a submatrix of the identity matrix.

The most straightforward way to compute $V_{\text {basis }}$ from Eq. (4) is to solve a problem of the form $A \boldsymbol{x}=\boldsymbol{b}_{\boldsymbol{i}}$ for each column of the matrix $B$. It would thus require solving $n_{s}$ systems of linear equations. For realistic conductor geometries, which have a large number of vertices, solving a single system may take up to a few minutes and solving $n_{s}$ systems quickly becomes impractical. Therefore, we propose the use of the adjoint method [23, which requires solving $n_{e}$ systems only. In the context of EMG, the number of electrodes is usually significantly smaller than the number of vertices in the muscle subdomain meshes, i.e. $n_{e}<<n_{s}$. Let us define $K=S A^{-1}$, which is a matrix of size $n_{e} \times n_{v}$. Because $A$ is symmetric, and the inverse of a symmetric matrix is also symmetric, we can write $K^{T}=A^{-1} S^{T}$. Then, $K$ can be found by solving the system: 


$$
A K^{T}=S^{T} .
$$

The matrix $S^{T}$ has $n_{e}$ columns and, thus, only $n_{e}$ linear systems need to be solved to find $K$. The basis solutions can then be found as:

$$
V_{\text {basis }}=K B
$$

\subsubsection{EMG signal of a single fiber activation}

The action potential generated by the flow of ionic currents across the muscle fiber membrane is the source of excitation. For a given intracellular action potential (IAP) model $V_{m}(z)$, the transmembrane current source per unit length is proportional to the second derivative of $V_{m}(z)$. A general description of the current density source traveling at velocity $v$ along the fiber with the origin at the neuromuscular junction at location $z_{0}$ is $[24,6,25]$ :

$$
\begin{aligned}
I(z, t)=\sigma_{i n} \pi r^{2} \cdot \frac{\partial}{\partial z}\left[\psi\left(z-z_{0}-v t\right)\right. & w_{L_{1}}\left(z-z_{0}-\frac{L_{1}}{2}\right)- \\
& \left.\psi\left(-z+z_{0}-v t\right) w_{L_{2}}\left(z-z_{0}+\frac{L_{2}}{2}\right)\right]
\end{aligned}
$$

where $z \in[0,1]$ is a location along the fiber, $\psi(z)=\frac{d}{d z} V_{m}(-z), L_{1}$ and $L_{2}$ are the semi-lengths of the fiber from the end-plate to the right and to the left tendon, respectively, $\sigma_{i n}$ is the intracellular conductivity, and $r$ is the fiber radius. We have chosen $w_{L}$ to be a Tukey window, as proposed in [26]. The IAP $V_{m}\left[\frac{m V}{m m}\right]$ can be mathematically described in the space domain as proposed in [27:

$$
V_{m}(z)=96 z^{3} e^{-z}-90 .
$$

Let $\boldsymbol{r}(z)$ be a parametrized fiber geometry. Combining the transfer function of a point source in Eq. (3) with the fiber's current density in Eq. (8), we obtain the equation for the EMG signal resulting from a single fiber activation:

$$
\boldsymbol{v}_{\text {fiber }}(t)=\int \boldsymbol{v}_{\text {point }}(\boldsymbol{r}(z)) I(z, t) d z .
$$

This integral can be efficiently approximated by discretizing the fiber geometry into sufficiently dense spatial samples $\left\{\boldsymbol{r}\left(z_{i}\right)\right\}_{i}$ and assuming that $\boldsymbol{v}_{\text {point }}(\boldsymbol{r}(z))$ is piecewise constant around these points. If we also rewrite Eq. (8) in a shorter form as $I(z, t)=\sigma_{i n} \pi r^{2} \cdot \frac{\partial}{\partial z} F(z, t)$, Eq. 99 becomes: 


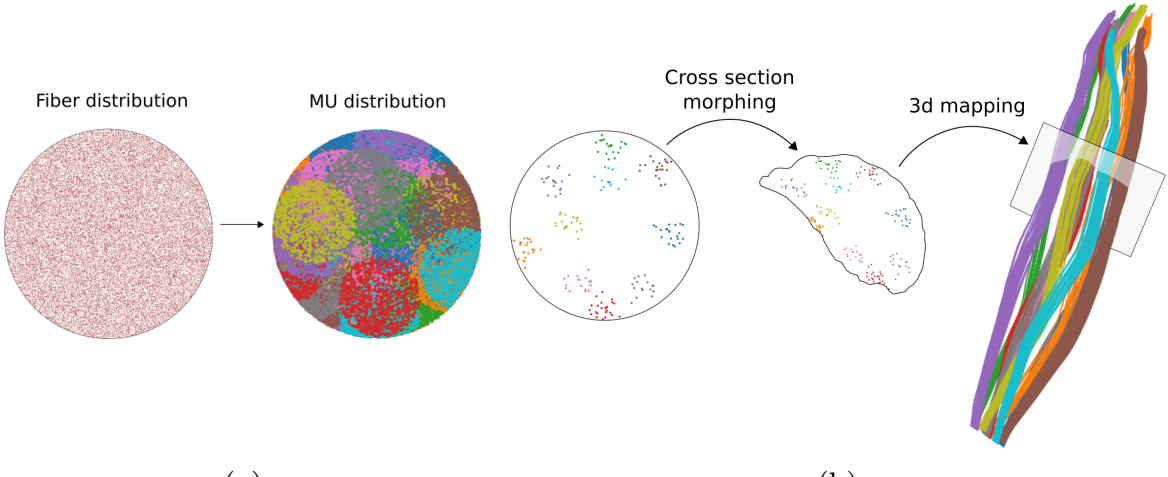

(a)

(b)

Figure 3: Fiber and motor unit distributions. (a) Uniformly distributed fibers inside a unit circle are grouped into motor units of different sizes, locations and territories. (b) Example of mapping of 10 small motor units from the circle into an arbitrary muscle by morphing the unit circle into the muscle cross section.

$$
\begin{aligned}
\boldsymbol{v}_{\text {fiber }}(t) \approx \sum_{i} \boldsymbol{v}_{\text {point }}\left(\boldsymbol{r}\left(z_{i}\right)\right) \int_{z_{i}-\Delta_{i}}^{z_{i}+\Delta_{i}} I(z, t) d z= \\
\quad \sum_{i} \boldsymbol{v}_{\text {point }}\left(\boldsymbol{r}\left(z_{i}\right)\right) \int_{z_{i}-\Delta_{i}}^{z_{i}+\Delta_{i}} \sigma_{i n} \pi r^{2} \cdot \frac{\partial}{\partial z} F(z, t) d z= \\
\sigma_{i n} \pi r^{2} \sum_{i} \boldsymbol{v}_{\text {point }}\left(\boldsymbol{r}\left(z_{i}\right)\right)\left(F\left(z_{i}+\Delta_{i}, t\right)-F\left(z_{i}-\Delta_{i}, t\right)\right) .
\end{aligned}
$$

Note, that $\boldsymbol{v}_{\text {point }}\left(\boldsymbol{r}\left(z_{i}\right)\right)$ can be efficiently computed from Eq. (5). Moreover, once $\boldsymbol{v}_{\text {point }}\left(\boldsymbol{r}\left(z_{i}\right)\right)$ are computed for all given fibers, we can change the parameters of the current source density (action potential waveform shape, propagation velocity, location of neuromuscular junction), and compute the corresponding EMG signal with Eq. 10 by only matrix multiplication complexity.

\subsection{Geometrical and physiological modeling of motor units}

The motor unit action potential (MUAP) is the summation of the single fiber action potentials (APs) of the muscle fibers in the MU. Different types of MUs can be modeled [28, 29. Our approach consists in generating fiber and motor unit distributions inside a unit circle, and then projecting it into arbitrary 3D muscle geometry (Fig. 3b), using methods similar to those described in [30]. This provides a high level of control for the fiber and MU distribution parameters independently of a particular muscle geometry. A common way to simulate fibers and MUs is to start by defining MU positions, sizes and territories, and then simulate fibers inside these MUs according to their parameters [31, 32. We, 
however, propose another approach. First, we simulate uniformly distributed fibers inside a unit circle. Then, MU centers and their circular territories are generated and, finally, we associate each fiber to an MU. A fiber is associated to one of the MUs that contains it inside its territory with a probability proportional to the MU density (Fig. 3a). This approach has two main advantages. First, it guaranties (by construction) the uniform fiber distribution inside a circular muscle cross-section. Second, once fibers are generated and projected into a muscle geometry, different MU distributions can be generated very quickly, without regenerating fibers and recomputing transfer functions $\boldsymbol{v}_{\text {point }}\left(\boldsymbol{r}\left(z_{i}\right)\right)$ for their nodes.

\section{MU recruitment model}

During muscle contraction, the MUs are recruited according to the size principle [33]. This can be simulated by associating a threshold of excitation to each MU, as described for example by Fuglevand et al. 34. Linear or non-linear rate coding models can be used [34, 35, 36.

The excitation rate as a function of time for each muscle is converted into the firing rates of the active MUs. Inter-discharge intervals are then generated with variability of the discharges around the mean firing interval 37 .

\section{Results}

The implementation of the main steps presented in the previous section can be summarized as follows. Once the matrices $S, A$ and $B$ are computed, the matrix $K$ is determined using Eq. (6) by solving $n_{e}$ linear systems. Then, Eq. (7) is used to find the solutions for $n_{s}$ basis points, which is a fast matrix multiplication operation. For any given point source location $\boldsymbol{r}$, we compute its barycentric coordinates in associated tetrahedron and apply Eq. (5) to get values of electrical potentials at electrode locations. Finally, for a given fiber geometry, the single fiber action potential as recorded by the EMG electrodes is computed using Eq. (9).

The results presented in this study are obtained using a Python implementation of the proposed strategy. Assembling the matrix $A$ and solving the system (6) is delegated to the FEniCS computing platform [38, 39. The forearm geometry that is here representatively used as a conductor model is taken from the website of BodyParts3D, The Database Center for Life Science 22]. The volume mesh is generated from the surface meshes of the forearm tissues using the CGAL C++ library [40].

\subsection{Comparison with an analytical solution}

First, we validated our numerical solution by comparing it with an analytical one (using the model presented in [7) for a simple volume conductor geometry. We used a four layer cylindrical model with layers corresponding to bone $(r=$ 


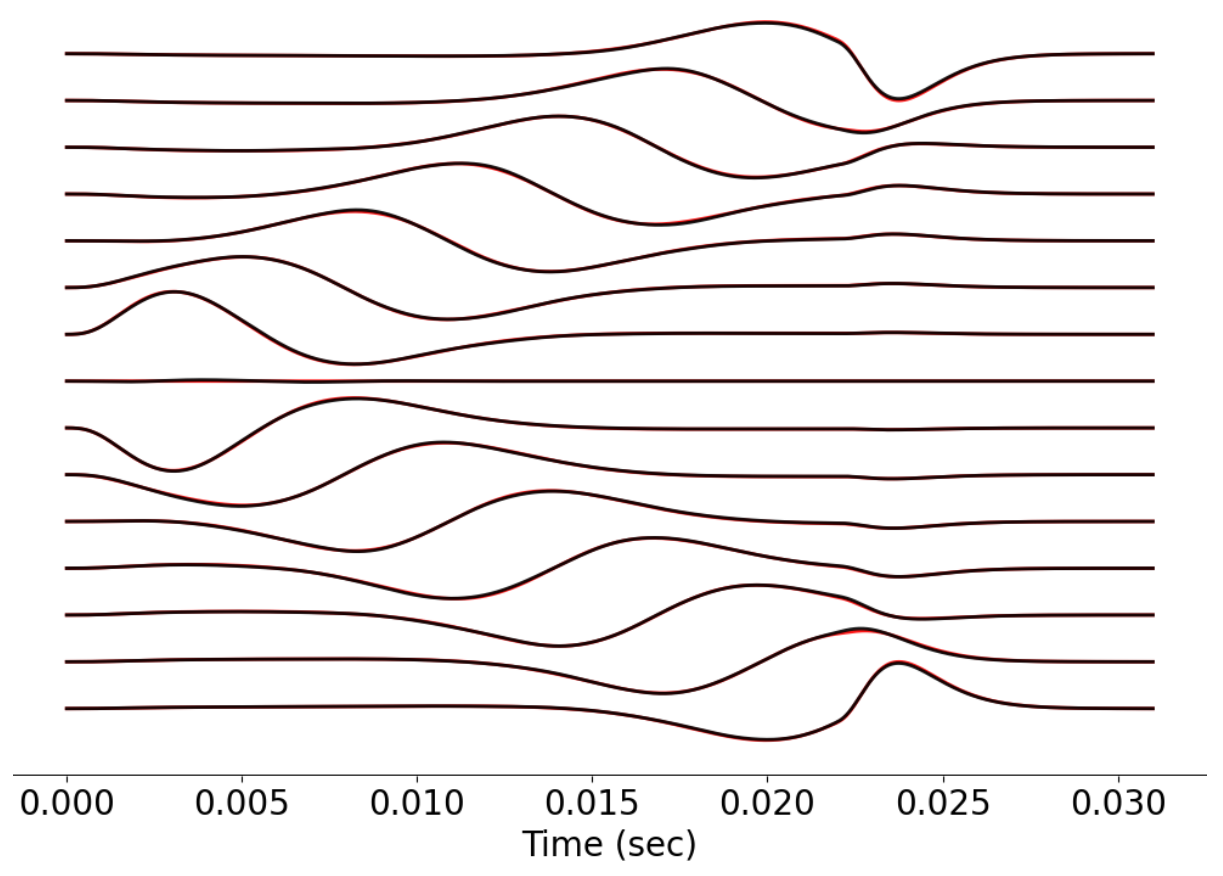

Figure 4: Comparison of the numerical and analytical [7] solutions for a four layer cylindrical volume conductor model: analytical (red) and numerical (black) sEMG signals for the differential electrode montage. The depth of the source fiber is $1 \mathrm{~mm}$ from the muscle surface.

$0.7 \mathrm{~cm})$, muscle $(r=2 \mathrm{~cm})$, fat $(r=2.3 \mathrm{~cm})$ and skin $(r=2.4 \mathrm{~cm})$ surfaces. 16 point electrodes were simulated right on top of a fiber. The fiber was located at varying depths into the muscle tissue, in the range $1 \mathrm{~mm}$ to $11 \mathrm{~mm}$. Differential sEMG signal was simulated using analytical and numerical solutions of the forward problem. The normalized mean square error between the two solutions depended on the depth of the fiber and varied between $3 \%$ (1mm depth from the muscle surface) and $5 \%$ (11mm depth).

Let us notice that the two volume conductor models in this validation are not exactly the same. The theoretical solution is computed for an infinitely long cylinder (repeated periodically when discretized), while the numerical solution uses a cylinder of a large (sufficiently longer than the fiber and the electrode array) yet finite length. Increasing the length of the cylinder did not significantly alter the error.

Fig. 4 shows the analytical (red) and numerical (black) solutions for the fiber depth of $1 \mathrm{~mm}$ from the muscle surface. Because of the low error value, the two waveforms are almost indistinguishable. 


\begin{tabular}{|c|c|c|c|c|}
\hline $\begin{array}{c}\text { General } \\
\text { basis points }\end{array}$ & $\begin{array}{c}\text { Fibers } \\
\text { basis points }\end{array}$ & $\begin{array}{c}\text { Fibers } \\
\text { EMG response }\end{array}$ & $\begin{array}{c}\text { MUs } \\
\text { EMG response }\end{array}$ & $\begin{array}{c}\text { Raw sEMG } \\
\text { assembling }\end{array}$ \\
\hline $\begin{array}{c}7 \mathrm{~min} \\
(13 \mathrm{sec} / \mathrm{elec})\end{array}$ & $2 \mathrm{~min}$ & $30 \mathrm{sec}$ & $0.8 \mathrm{sec}$ & $2.6 \mathrm{sec}$ \\
\hline
\end{tabular}

Table 1: Computational performance of each of the main steps of a raw EMG simulation. General basis points computation refers to equation (7); fiber basis points are computed with equation (5); fibers EMG response is computed with equation (9).

\subsection{Computational performance}

In this section we report the computational time of the proposed model for a specific simulation case. Note, that no multiprocessing tools were used in these computations. Each step, however, is highly scalable and can be efficiently distributed between parallel processes, which would significantly increase the performance. Computations for each muscle and fiber are independent and can be performed in parallel. Parallel computing would also apply to the electrodes in the general basis points computation.

For the purpose of demonstration, we simulated a 1-min long, $100 \%$ maximum voluntary contraction (MVC) excitation of the Brachioradialis muscle with 50000 individual fibers and 200 motor units. The mesh of the volume conductor contained $2.1 \mathrm{M}$ vertices which formed 13M tetrahedra. 16 rectangular and 16 circular electrodes were included in the model. The sampling frequency of the simulated signals was $2000 \mathrm{~Hz}$. Table 1 shows the computational time for each of the main steps in this simulation.

Note that a list of parameters need to be provided for each step of Table 1 However, an important property of our model is that each step depends only on the data produced by the previous steps. This property can be exploited to change some simulation parameters without recomputing every step of the simulation. For example, it is not necessary to recompute solutions for the fiber basis points if only the simulation parameters related to the MU distribution or recruitment model are modified. In this example, the total simulation time for this new set of parameters will only take approximately $0.8+2.6=3.4 \mathrm{~s}$.

A brief description of the main parameters required at each step follows. The full arm and electrode geometry as well as the tissue conductivities define the computation general basis points. To compute fibers basis points solutions, the 3D geometry of the fibers is required. Computing the fiber EMG responses requires the shape of the intracellular AP waveforms, AP propagation velocity, sizes of tendon and active fiber parts, neuromuscular junction location, fiber diameter and intracellular conductivity, and sampling frequency. To compute the MUs action potentials, the MU distribution in the muscle, i.e. the association of fibers to each motor unit, need to be defined. In the proposed model, once the number of MUs, their sizes and territory areas are selected, the MU distribu- 


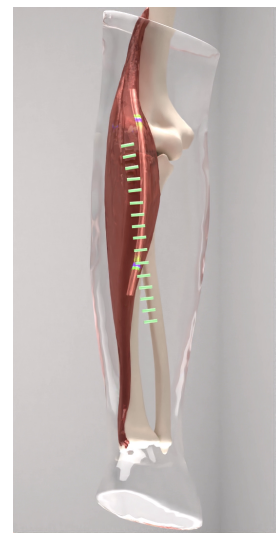

(a)

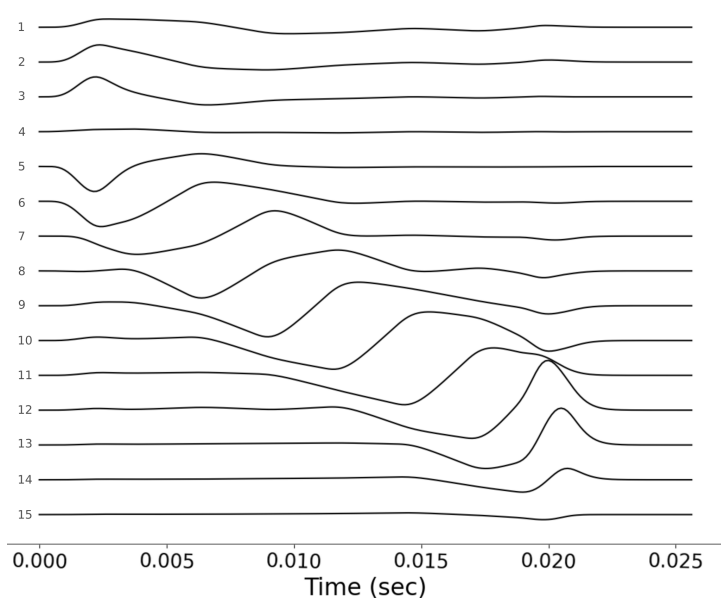

(b)

Figure 5: Single fiber activation in the Brachioradialis muscle: (a) Fiber and electrodes geometry. (b) Differential sEMG signal of a single fiber activation.

tion is randomly generated. Finally, to synthesize the sEMG signal, the muscle excitation drives and recruitment model parameters (motor unit recruitment thresholds and firing rates) are required.

\subsection{Simulation examples}

\section{Single fiber activation}

We begin by simulating the EMG signal associated to a single fiber activation inside the Brachioradialis muscle. The signal for an array of 16 rectangular electrodes and $8 \mathrm{kHz}$ time resolution was generated. Fig. 5 illustrates the geometrical location of the fiber and the corresponding sEMG signal in differential electrode montage. Different distinctive features are present in the simulated signal that are also observed in experimental sEMG signals 41. In particular, electrodes of the channel 4 are located on different sides of the neuromuscular junction (NMJ) and thus cancel each other out. Channels 7-11 contain propagating sEMG components resulted from the fiber AP propagating from the NMJ to the tendons. Channels 2-6, as well as channels 12-15, contain nonpropagating sEMG components, which are due to the AP generation at the NMJ and its extinction at the tendon (end-of-fiber effect), respectively.

\section{Single muscle activation}

The next example is a simulation of an excitation of a single muscle (Fig. 6). A simple excitation drive for the Brachioradialis muscle was simulated. It gradually goes from $0 \%$ to $100 \%$ maximum voluntary contraction and back to 0 . $50 \mathrm{k}$ muscle fibers were generated inside the muscle and distributed within 200 motor 


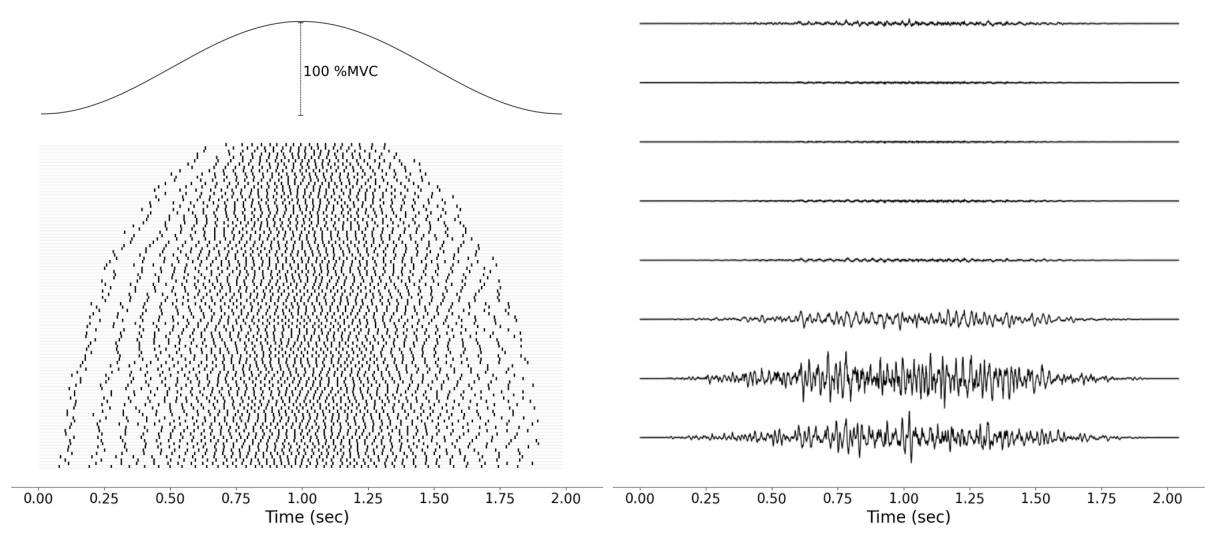

Figure 6: Activation of the Brachioradialis muscle. Muscle excitation is first decomposed into motor unit impulse trains (on the left). Then, the sEMG signal recorded by 8 bipolar electrodes (see Fig. 2a) was simulated (on the right).

units. The size of MUs varied exponentially from 11 to 1150 fibers. The areas of MU territories varied from $10 \%$ to $50 \%$ of the muscle cross-sectional area. The muscle excitation drive was decomposed into MU impulse trains according to the size principle. In this example, the firing rate for each $\mathrm{MU}$ ranged from 8 $\mathrm{Hz}$ to $35 \mathrm{~Hz}$ and all MUs were recruited when an excitation level of $75 \% \mathrm{MVC}$ was reached.

\section{Multiple muscle coordination}

Finally, we simulated sEMG signals from multiple muscle excitations corresponding to wrist flexion and extension (Fig. 8). Two groups of muscles were involved. The flexor group included the Palmaris longus, Flexor carpi ulnaris (ulnar head), Flexor carpi ulnaris (humeral head), and Flexor carpi radialis muscles. The extensor group included the Extensor digitorum, Extensor carpi ulnaris, Extensor carpi radialis brevis, and Extensor carpi radialis longus muscles. During a wrist flexion, the muscles of the flexor group reached an excitation level of 70-90\% MVC, while the extensor muscles acted as antagonists with excitation in the range 10-30\% MVC. During extension, the agonist-antagonist roles switched. Moreover, a small but constant excitation of the abduction muscle group was added to simulate the wrist resistance against gravity. The abduction muscle group included the Flexor carpi radialis, Extensor carpi radialis brevis, and Extensor carpi radialis longus muscles. For each muscle, a number of muscle fibers between $32 \mathrm{k}$ and $78 \mathrm{k}$ was simulated, depending on the muscle cross sectional area. Muscle fibers were distributed within motor units, whose number varied from 150 to 300 per muscle.

Fig. 8 clearly shows the similarities in signal characteristics between experimental and simulated data. Beside the different activation across the electrodes during flexion and extension, the effect of wrist abduction is also visible in both 


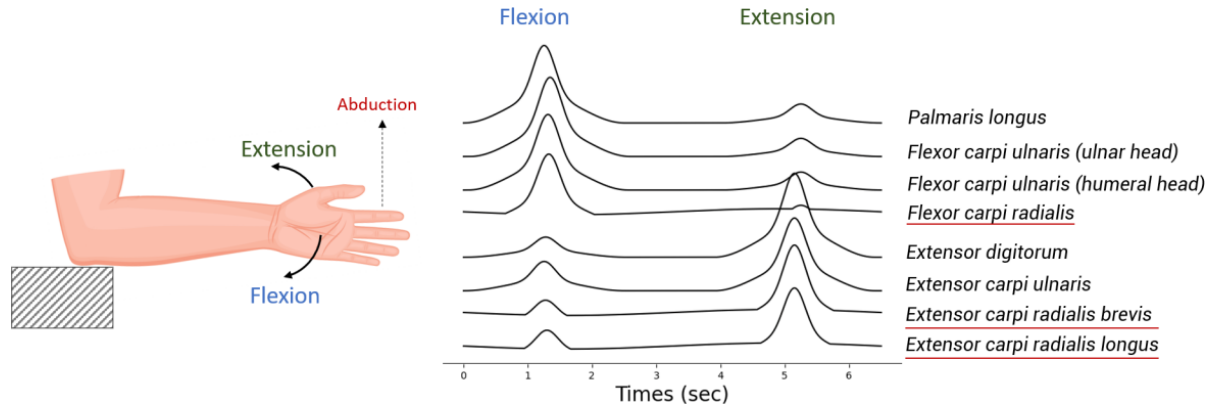

Figure 7: Muscle excitation model for wrist flexion/extension. During a wrist flexion, the muscles of the flexor group reached an excitation level of 70-90\% MVC, while the extensor muscles acted as antagonists with excitation in the range 10-30\% MVC. During extension the roles were switched. A constant wrist abduction was also added to simulate wrist resistance against gravity to keep it in the horizontal position.
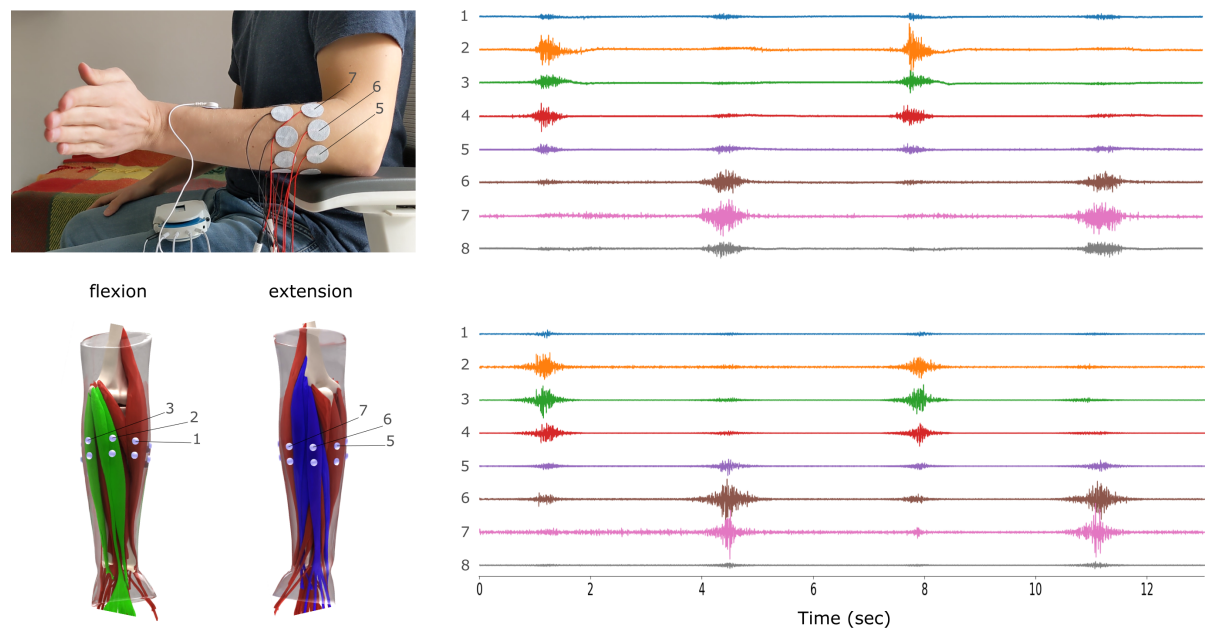

Figure 8: Comparison of experimental (top) and simulated (bottom) sEMG signals for the left wrist flexion and extension. The experimental signals were measured with 8 bipolar electrodes located around the forearm. For simulation, the flexor (green) and extensor (blue) muscle groups were activated in turn with activation peaks aligned with the experimental signal peaks.

data sets. Thus, channel 7 presents signal activity during the whole duration of the simulation, with peak of the signal during extension.

However, as the model used for this simulation is not personalized, simulated signals do not perfectly replicate all the details of the experimental signals. For example, channel 8 for the experimental measurements has remarkably higher amplitude during extension than in the simulated conditions. 

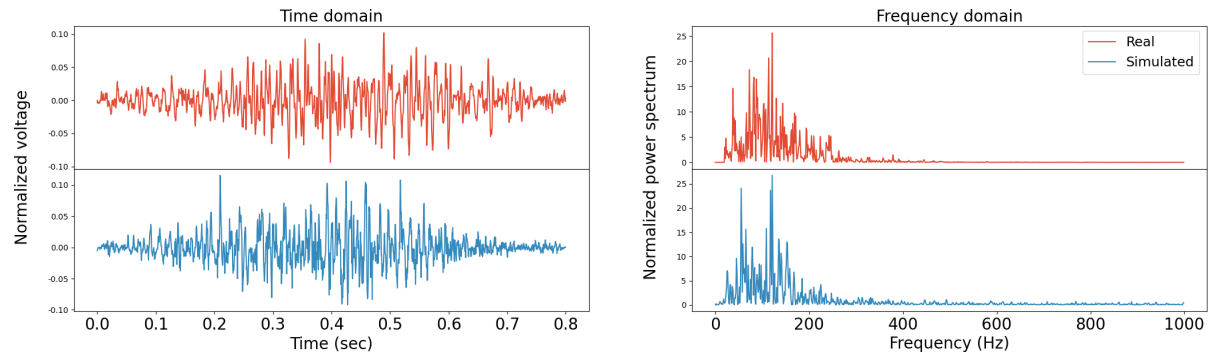

Figure 9: Comparison of experimental (red) and simulated (blue) single channel normalized sEMG signals in the time and frequency domains.

In addition to the analysis in time domain, simulated data were compared against the experimental data in the frequency domain. Fig. 9 shows an example of the measured and simulated single channel sEMG. The two signals have similar power spectra. It has to be noted that the spectral characteristics of a signal strongly depends on multiple simulation parameters. In this example, we run several hundreds simulations by varying the simulation parameters in a realistic range and selected the set of parameters leading to the minimal spectral difference. This approach, which is a simple version of inverse modelling, was possible because of the extremely high computational speed of the simulations.

\section{Discussion}

We have proposed an efficient computational approach to solve the volume conductor problem in the generation of surface EMG signals. The method provides the solution to the generation of EMG signals from realistic volume conductor properties and number of muscle fibers, within limited computational time. Moreover, once the model is solved for a specific volume conductor, the proposed approach provides solutions for an arbitrary number and properties of fibers and motor units, activations of the motor units as well as signal duration and sampling frequency, without re-computing the volume conductor solution. This property allows us to generate EMG signals within a computational time compatible with real-time signal generation (see example videos on https://www . youtube .com/channel/UCulDYbGBvSkJzaPlFgzKQ2Q). The proposed model is the only available EMG simulator with realistic description of the volume conductor and optimized for such computational efficiency. The modelling of EMG signals is based on the description of the electrical activity of the fiber membrane, in terms of intracellular and transmembrane electrical potentials. The electric field generated by the fiber electrical activity is recorded in a volume conductor, which is often described under the electrostatic assumption. Various mathematical descriptions of the intracellular action potential and volume conductor have been previously provided [27, 25, 42. The computational efficiency in the volume conductor solution has been recognized as an 
important component of EMG modelling and some attempts to decrease the computational time in EMG simulations have been described. For example, Dimitrov \& Dimitrova [43] substantially decreased the computational time in EMG modeling by computing the impulse response of the volume conductor, so that the surface action potentials generated by muscle fibers were determined as a convolution between the first derivative of the intracellular action potential and the impulse response. This approach, based on the classic description of a single fiber electrical activity by Lorente de Nó [44, allowed to compute one single convolution to generate extracellular potentials generated by an arbitrary analytical description of the intracellular action potential, with generation at the end plate, propagation along the fiber, and extinction at the tendons. However, the volume conductor in the model proposed by Dimitrov \& Dimitrova 43 was an infinite homogeneous medium, which limited the accuracy of the simulations with respect to realistic conditions.

The mathematical description of the full generation and extinction process of the intracellular action potential by the first temporal derivative, as proposed by Dimitrov \& Dimitrova [43], provided an analytical method to describe the source of EMG signal with one single spatio-temporal function. In principle, this description can be used with complex volume conductors as long as a transfer function can be computed. Farina et al. proposed a spatial frequency domain description of non-homogeneous planar [6] and cylindrical [7] volume conductors, so that the effect of the volume conductor could be described by a temporal convolution with the first derivative of the intracellular action potential, as previously done with simpler geometries by Dimitrov \& Dimitrova [43. The same approach could be applied to numerical descriptions of the volume conductor when the property of spatial invariance along the direction of propagation of the muscle fibers was satisfied [5]. These solutions therefore were restricted to cylindrical volume conductors, i.e. volume conductor with invariant cross-section along the fiber direction.

Realistic models using numerical solutions have also been recently proposed. The previous most complete and efficient model has been proposed by Pereira Botelho et al. [15. These authors have used an anatomically accurate model to simulate EMG signals generated during index finger flexion and abduction. They gained computational speed by using the principle of reciprocity. In fact, one part of our calculations also includes the adjoint method which is an algebraic representation of this principle. By reciprocity, Pereira Botelho et al. [15] reported a computational time of 1 hour for simulating the activation of nearly 15500 fibers for 5 electrodes. This time remains impractical for simulating arbitrary large data sets for a variety of parameter values. The model we proposed in this paper substantially surpasses the computational efficiency reported in 15. (see our Table 1, as an example). To achieve this extreme reduction in computational time without any constraints on the volume conductor, we have optimized the numerical computation by reducing the main calculations to the solutions corresponding to basis points, from which a general solution can be obtained. The approach does not only reduce the computational time for a full simulation but also allows us to scale the solution, so that new solutions for 
the same volume conductor can be obtained without re-computing the volume conductor transformation. In this way, the generation of EMG signals within the same volume conductor, but varying all other simulation parameters, can be performed extremely fast. Complex EMG signals from tens of thousands of muscle fibers located in multiple muscles, can therefore be generated (and regenerated with different parameter values) in a computational time of the order of seconds. The described model is the first that allows an extremely accurate signal generation within a limited computational time. Contrary to previous models, the proposed simulator does not compromise accuracy and computational speed.

In perspective, to make simulated sEMG signals even more realistic, the current model can be extended by including advanced noise and artifacts modeling, biomechanical modeling of the musculoskeletal system and dynamic volume conductor and fiber geometry. While these aspects are beyond the scope of this paper, they are relevant features to include in future developments.

One of the reasons for developing a model with high accuracy and speed, is its potential for addressing the inverse problem, i.e. to identify the location of active motor units or muscle compartments within the volume conductor given the recorded EMG signals at the skin surface. The identification of model parameters in inverse modelling requires the fast computation of a large number of solutions for the identification of a globally optimal solution. Current attempts to EMG inverse modelling are based on simplified volume conductors, as well as simplified assumptions in terms of motor unit activation, in order to identify the inverse solution in a realistic time 45. The model proposed in this work removes all the simplifications to realistic simulations, maintaining high computational speed. Another application of precise and fast EMG simulations is data augmentation in AI-based signal classification and/or decomposition. The proposed model can indeed be used for generating a large variability of data from multiple volume conductor and fiber properties in order to generalize processing methods across experimental sessions and individuals.

In conclusion, we have proposed a fast and highly accurate approach to simulate surface EMG signals. The computational efficiency of our model greatly surpasses any other currently available solution. The modelling approach is based on an efficient determination of the EMG solution by a modular approach for which processing steps do not need to be repeated if some of the simulation conditions remain constant. The model has the potential of substantially expanding the applications of EMG modelling, especially in relation to modern AI-based approaches of inverse modelling and signal decomposition.

\section{Acknowledgments}

DF is funded by the European Research Council (project NaturalBionicS) and the EPSRC (project NISNEM). 


\section{Competing interests}

KM and SDG are founders of Neurodec company.

\section{References}

[1] Dick F. Stegeman, Joleen H. Blok, Hermie J. Hermens, and Karin Roeleveld. Surface emg models: Properties and applications, 2000.

[2] Robert Plonsey. Action potential sources and their volume conductor fields. Proceedings of the IEEE, 65:601-611, 1977.

[3] Robert Plonsey and Dennis B. Heppner. Considerations of quasistationarity in electrophysiological systems. The Bulletin of mathematical biophysics, 29(4):657-664, December 1967.

[4] Anco Heringa, Dick F. Stegeman, Gèrard J.H. Uijen, and Johannes P.C. De Weerd. Solution methods of electrical field problems in physiology. IEEE Transactions on Biomedical Engineering, BME-29, 1982.

[5] Dario Farina, Luka Mesin, and Simone Martina. Advances in surface electromyographic signal simulation with analytical and numerical descriptions of the volume conductor. Medical and Biological Engineering and Computing, 42, 2004 .

[6] Dario Farina and Roberto Merletti. A novel approach for precise simulation of the emg signal detected by surface electrodes. IEEE Transactions on Biomedical Engineering, 48, 2001.

[7] Dario Farina, Luca Mesin, Simone Martina, and Roberto Merletti. A surface emg generation model with multilayer cylindrical description of the volume conductor. IEEE Transactions on Biomedical Engineering, 51, 2004.

[8] Joleen H. Blok, Dick F. Stegeman, and Adriaan Van Oosterom. Three-layer volume conductor model and software package for applications in surface electromyography. Annals of Biomedical Engineering, 30, 2002.

[9] Theis H. J. M. Gootzen, Dick F. Stegeman, and Adriaan van Oosterom. Finite limb dimensions and finite muscle length in a model for the generation of electromyographic signals. Electroencephalography and Clinical Neurophysiology/ Evoked Potentials, 81, 1991.

[10] Andrew J. Fuglevand, David A. Winter, Aftab E. Patla, and Daniel Stashuk. Detection of motor unit action potentials with surface electrodes: influence of electrode size and spacing. Biological Cybernetics, 67, 1992.

[11] Dick F. Stegeman and Wim H.J.P. Linssen. Muscle fiber action potential changes and surface emg: A simulation study. Journal of Electromyography and Kinesiology, 2, 1992. 
[12] Guang Yue, Andrew J. Fuglevand, Michael A. Nordstrom, and Roger M. Enoka. Limitations of the surface electromyography technique for estimating motor unit synchronization. Biological Cybernetics, 73, 1995.

[13] Karin Roeleveld, Joleen H. Blok, Dick F. Stegeman, and Adriaan Van Oosterom. Volume conduction models for surface emg; confrontation with measurements. volume 7, 1997.

[14] Jochen Schneider, Jiri Silny, and Günter Rau. Influence of tissue inhomogeneities on noninvasive muscle fiber conduction velocity measurements - investigated by physical and numerical modeling. IEEE Transactions on Biomedical Engineering, 38, 1991.

[15] Diego Pereira Botelho, Kathleen Curran, and Madeleine M. Lowery. Anatomically accurate model of emg during index finger flexion and abduction derived from diffusion tensor imaging. PLoS Computational Biology, $15,2019$.

[16] Alexander Kenneth Clarke, Seyed Farokh Atashzar, Alessandro Del Vecchio, Deren Barsakcioglu, Silvia Muceli, Paul Bentley, Filip Urh, Ales Holobar, and Dario Farina. Deep learning for robust decomposition of high-density surface emg signals. IEEE Transactions on Biomedical Engineering, 68, 2021.

[17] Ales Holobar and Dario Farina. Non-invasive neural interfacing with wearable muscle sensors. IEEE Signal Processing Magazine, in-press 2021.

[18] Luca Mesin. Inverse modelling to reduce crosstalk in high density surface electromyogram. Medical Engineering and Physics, 85, 2020.

[19] Charles Nicholson and John A. Freeman. Theory of current source density analysis and determination of conductivity tensor for anuran cerebellum. Journal of Neurophysiology, 38, 1975.

[20] Klas H. Pettersen, Henrik Lindén, Anders M. Dale, and Gaute T. Einevoll. Extracellular spikes and current-source density, pages 92-135. Cambridge University Press, Cambridge, UK, 2010.

[21] Lutz Angermann Peter Knabner. The Finite Element Method for the Poisson Equation, pages 46-91. Springer New York, New York, NY, 2003.

[22] MS Windows NT kernel description.

[23] Sylvain Vallaghé, Théodore Papadopoulo, and Maureen Clerc. The adjoint method for general eeg and meg sensor-based lead field equations. Physics in Medicine and Biology, 54, 2009.

[24] Roberto Merletti and Dario Farina. Surface Electromyography: Physiology, Engineering and Applications. 2016. 
[25] Robert Plonsey. The active fiber in a volume conductor. IEEE Transactions on Biomedical Engineering, BME-21, 1974.

[26] Vincent Carriou, Sofiane Boudaoud, Jeremy Laforet, and Fouaz Sofiane Ayachi. Fast generation model of high density surface emg signals in a cylindrical conductor volume. Computers in Biology and Medicine, 74, 2016.

[27] Poul Rosenfalck. Intra- and extracellular potential fields of active nerve and muscle fibres. a physico-mathematical analysis of different models. Acta physiologica Scandinavica. Supplementum, 321:1-168, 1969.

[28] R. E. Burke, D. N. Levine, P. Tsairis, and F. E. Zajac. Physiological types and histochemical profiles in motor units of the cat gastrocnemius. The Journal of Physiology, 234, 1973.

[29] Stefano Schiaffino and Carlo Reggiani. Fiber types in mammalian skeletal muscles. Physiological Reviews, 91, 2011.

[30] Luca Modenese and Josef Kohout. Automated generation of threedimensional complex muscle geometries for use in personalised musculoskeletal models. Annals of Biomedical Engineering, 48, 2020.

[31] Kevin G. Keenan, Dario Farina, Roberto Merletti, and Roger M. Enoka. Influence of motor unit properties on the size of the simulated evoked surface emg potential. Experimental Brain Research, 169, 2006.

[32] Vincent Carriou, Jeremy Laforet, Sofiane Boudaoud, and Mariam Al Harrach. Realistic motor unit placement in a cylindrical hd-semg generation model. volume 2016-October, 2016.

[33] Elwood Henneman. Relation between size of neurons and their susceptibility to discharge. Science, 126, 1957.

[34] Andrew Fuglevand, David A. Winter, and Aftab E. Patla. Models of recruitment and rate coding organization in motor-unit pools. Journal of Neurophysiology, 70, 1993.

[35] Fouaz Sofiane Ayachi, Sofiane Boudaoud, and Catherine K Marque. Evaluation of muscle force classification using shape analysis of the semg probability density function: A simulation study. Medical and Biological Engineering and Computing, 52, 2014.

[36] Carlo J. De Luca and Emily C. Hostage. Relationship between firing rate and recruitment threshold of motoneurons in voluntary isometric contractions. Journal of Neurophysiology, 104, 2010.

[37] Todor I. Arabadzhiev, Vladimir G. Dimitrov, Nonna A. Dimitrova, and George V. Dimitrov. Influence of motor unit synchronization on amplitude characteristics of surface and intramuscularly recorded emg signals. European Journal of Applied Physiology, 108, 2010. 
[38] Anders Logg, Kent Andre Mardal, and Garth N. Wells. Automated solution of differential equations by the finite element method. Lecture Notes in Computational Science and Engineering, 84 LNCSE, 2012.

[39] The fenics project version 1.5. The FEniCS Project Version 1.5, 3, 2015.

[40] The CGAL Project. CGAL User and Reference Manual. CGAL Editorial Board, 5.2.1 edition, 2021.

[41] R. Merletti and S. Muceli. Tutorial. surface emg detection in space and time: Best practices. Journal of Electromyography and Kinesiology, 49, 2019 .

[42] Willemien Wallinga, S. L. Meijer, M. J. Alberink, M. Vliek, E. D. Wienk, and D. L. Ypey. Modelling action potentials and membrane currents of mammalian skeletal muscle fibres in coherence with potassium concentration changes in the t- tubular system. European Biophysics Journal, 28, 1999.

[43] George V. Dimitrov and Nonna A. Dimitrova. Precise and fast calculation of the motor unit potentials detected by a point and rectangular plate electrode. Medical Engineering and Physics, 20, 1998.

[44] Rafael Lorente de Nó. A study of nerve physiology. Studies from the Rockefeller institute for medical research. Reprints. Rockefeller Institute for Medical Research, 131, 1947.

[45] Yasuhiro Nakajima, Saran Keeratihattayakorn, Satoshi Yoshinari, and Shigeru Tadano. An emg-ct method using multiple surface electrodes in the forearm. Journal of Electromyography and Kinesiology, 24, 2014. 